\title{
Test Results for a Linear Tapered Slot Antenna with Varying Slot-Line Lengths and Mounting Configurations
}

\author{
Janice C. Rock Chris A. Hamner Perry Vanderford \\ United States Army Aviation and Missile Command \\ ATTN: AMSAM-RD-MG-RF \\ Redstone Arsenal, Alabama 35898 \\ 256-876-1426 \\ janice.rock@rdec.redstone.army.mil
}

\begin{abstract}
This paper considers two different mounting options for a Linear Tapered Slot Antenna (LTSA). Five different antenna designs, utilizing five different slot-line lengths, were tested. The primary purpose of the test was to explore the possibility of using this type of antenna as a radar-receiving structure for possible applications in guided, mini-projectiles. The main emphasis of the testing was to demonstrate the effects of mounting the antennas on a conducting surface.
\end{abstract}

\section{TABLE OF CONTENTS}

1. INTRODUCTION AND BACKGROUND

2. Test Procedures. 1

3. TEST RESULTS............................................................. 2

4. CONCLUSIONS ............................................................................ 5

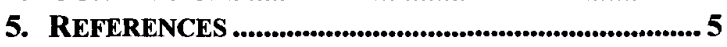

BIOGRAPHIES ........................................................................... 6

\section{INTRODUCTION AND BACKGROUND}

A series of Ka-Band antennas were produced for evaluation as candidates for mounting on a conducting surface. The Linear Tapered Slot Antenna (LTSA) is known as an endfire antenna because its maximum radiation is directed along the main axis. These antennas were designed with different slot line lengths that varied the antenna's input impedance and resulting voltage standing wave ratio (VSWR). The design work for the antennas can be found in Smith [1]. The emphasis of the testing was to determine the suitability of the antenna for use in guided mini-projectiles. The antenna would be mounted on the outside of the projectile and would be in direct contact with the surface of the projectile body. Because the E-field of the LTSA is created in the slot and would be parallel to any mounting surface, the radiation pattern measurements were collected with the antennas mounted on two different surfaces-one conducting and one simulating free-space. This was done to determine changes in the antenna patterns due to degradation of the E-field. Patterns were measured in an anechoic compact range chamber while the antennas were mounted on a sixinch by six-inch metal plate to simulate antenna mounting on the metal surface and then mounted on a small polystyrene block to simulate antenna mounting in air as a reference measurement. It was decided to use a coaxial line feed across the slot line of the antennas as the feed mechanism to the antenna for testing purposes.[2]

This feed is easy to prototype and low cost, but is not a normal feed technique at Ka-Band for the LTSA. Usually a microstrip feed or a waveguide probe feed structure would be utilized. The use of a coaxial line feed technique, while simple to build for this test, probably reduced input efficiency and thus the antenna gain. Although the most important aspect used to evaluate these candidate antennas are their respective radiation patterns, for further evaluation, their respective VSWR values were measured in the laboratory using a network analyzer. These test procedures are detailed in section II and the results presented in section 3 .

\section{Test Procedures}

Five different designs of the LTSAs were developed using different slot line lengths. By varying the slot line length $\left(\mathrm{L}_{\text {slot }}\right)$, the VSWR of the antenna can be adjusted. Our designs varied the slot line length by using five different lengths for $L_{e n d}$ as shown in Figure 1.

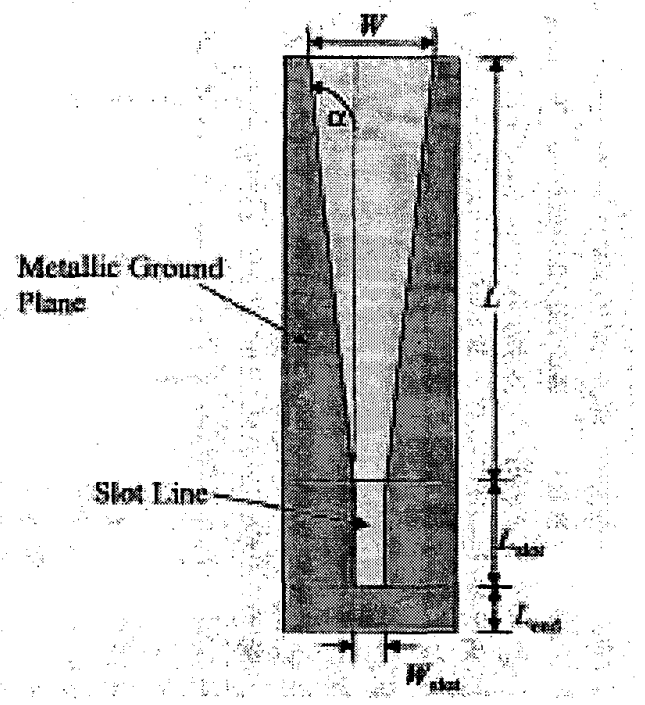

Figure 1. Definitions for a Linear Tapered Slot Antenna 
The slot line length was varied by changing $L_{\text {end }}$ from $0 \mathrm{~mm}$ to $4 \mathrm{~mm}$ in $1 \mathrm{~mm}$ increments. The coaxial feed was placed over the slot line at a distance of approximately $2 \mathrm{~mm}$ (this distance is close to $\lambda / 4$ and its use is a known impedance matching technique) from the bottom end of $\mathrm{L}_{\text {slot }}$ and is illustrated in Figure 2.
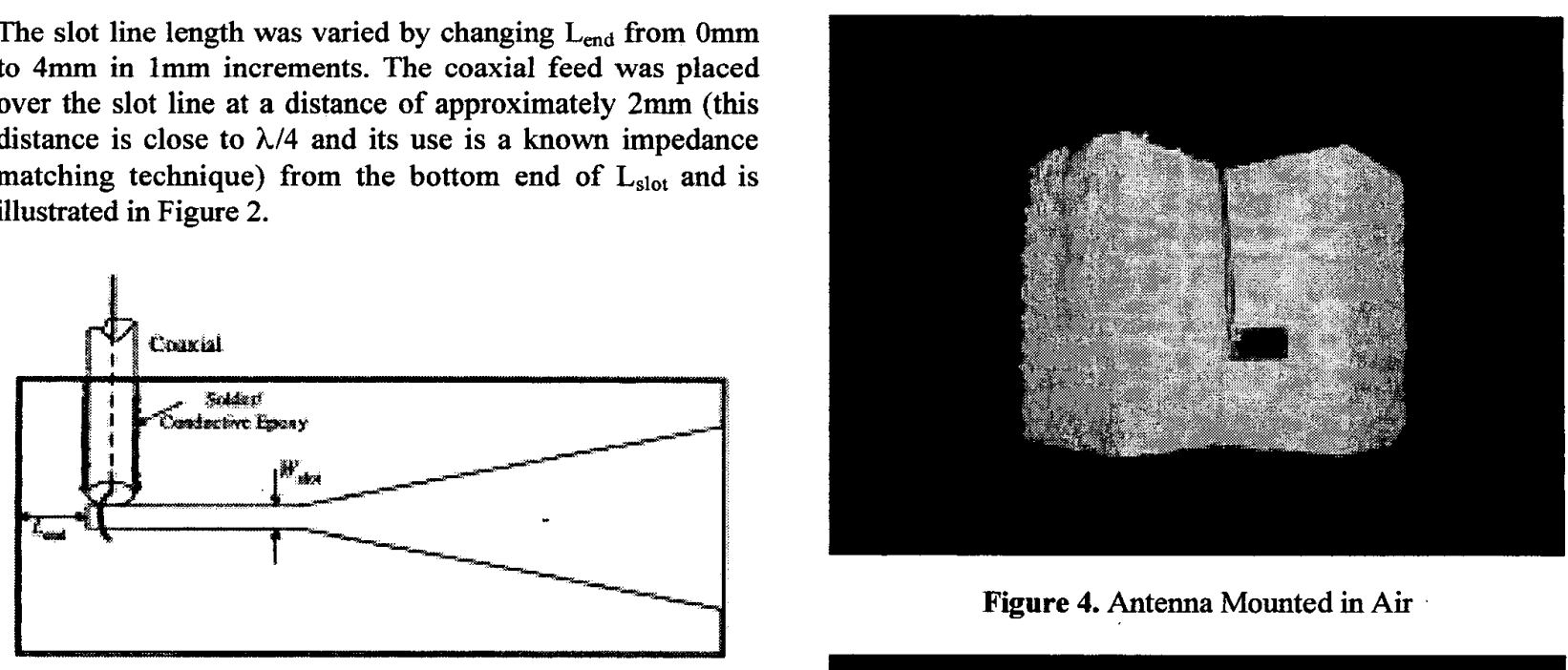

Figure 4. Antenna Mounted in Air

Figure 2. Coaxial Connection to the Antenna

Figure 3 shows the five different antennas that were used throughout the testing procedures.
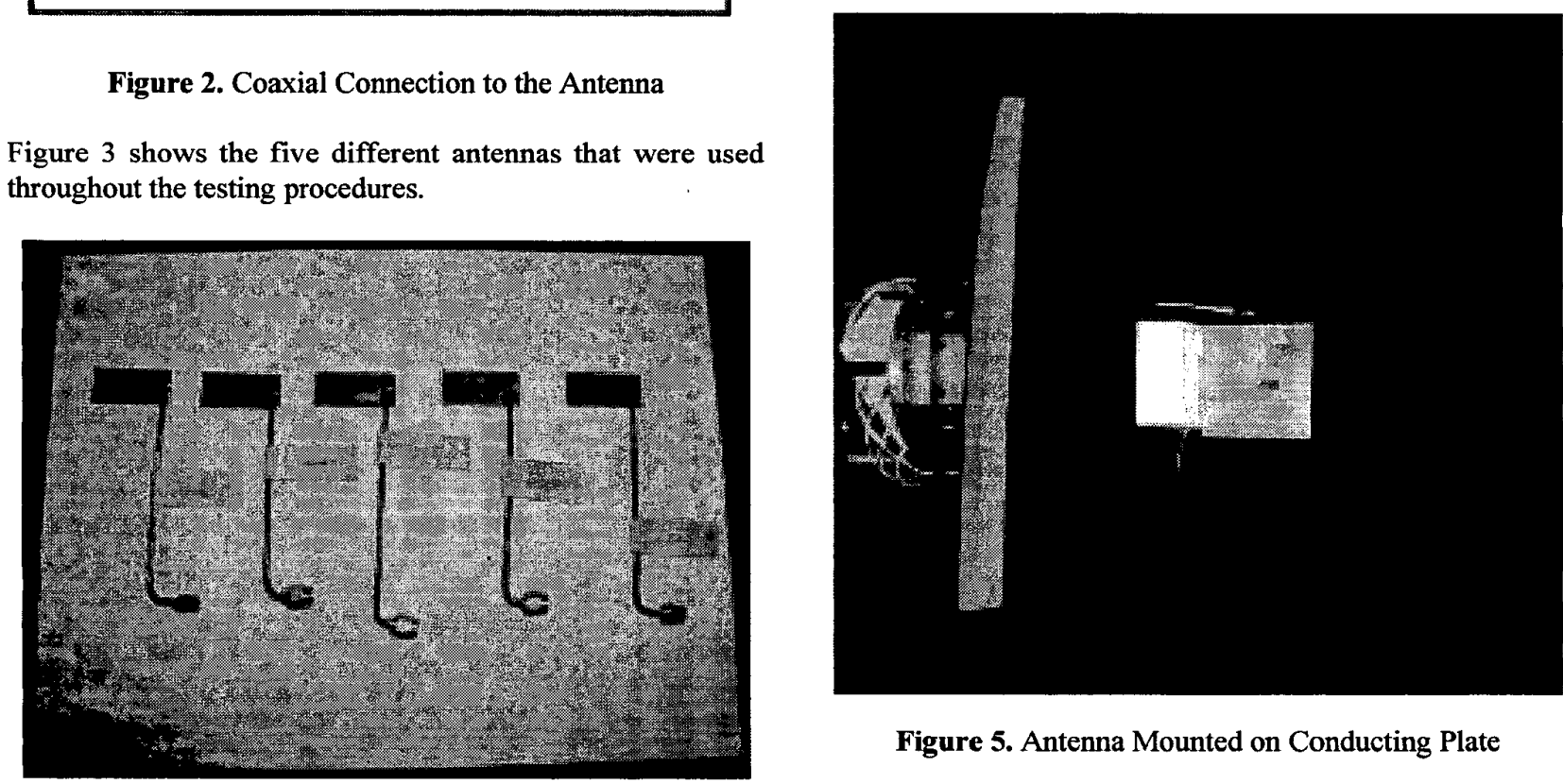

Figure 5. Antenna Mounted on Conducting Plate

Figure 3. Coaxially fed Linear Tapered Slot Antenna Designs

The antennas were mounted in two different configurations for testing, with both having a vertical polarization (Figure 4). First, they were mounted on a polystyrene base to simulate mounting in air. Azimuthal cuts were made for both co-polarization and cross-polarization orientations. While the compact range was configured for copolarization, full raster scans were completed for all five antenna designs.

Next, the antennas were mounted on a conductive base consisting of a $6 " \times 6$ " aluminum plate. For mounting configurations, metal plate and polystyrene only, anechoic material was placed between the compact range positioner and the antenna itself. The above-mentioned scans were repeated for this mounting configuration (Figure 5).

The antenna measurements were made using a compact range and Scientific Atlanta test equipment. The azimuthal cuts were made while moving the compact range positioner from $-135^{\circ}$ to $90^{\circ}$. The antennas were mounted so that the end fire position faced the compact range reflector at $0^{\circ}$ azimuth. At $-90^{\circ}$, the antenna was broadside (metallic face) to the reflector and at $90^{\circ}$, the antenna was once again broadside with the duroid facing the reflector. Input impedance measurements for all five designs were made using an Agilent 8722ES S-Parameter Network Analyzer.

\section{Test Results}

The resulting design beamwidth was $28^{\circ}$ to $44^{\circ}$. The anticipated mainbeam was in the endfire to near endfire direction. Most of the co-polarization patterns in air were not well formed. The $L_{\text {end }}=2$ design gave the most desirable pattern and is included in the Figures 6-11. 
Vol. 2-935

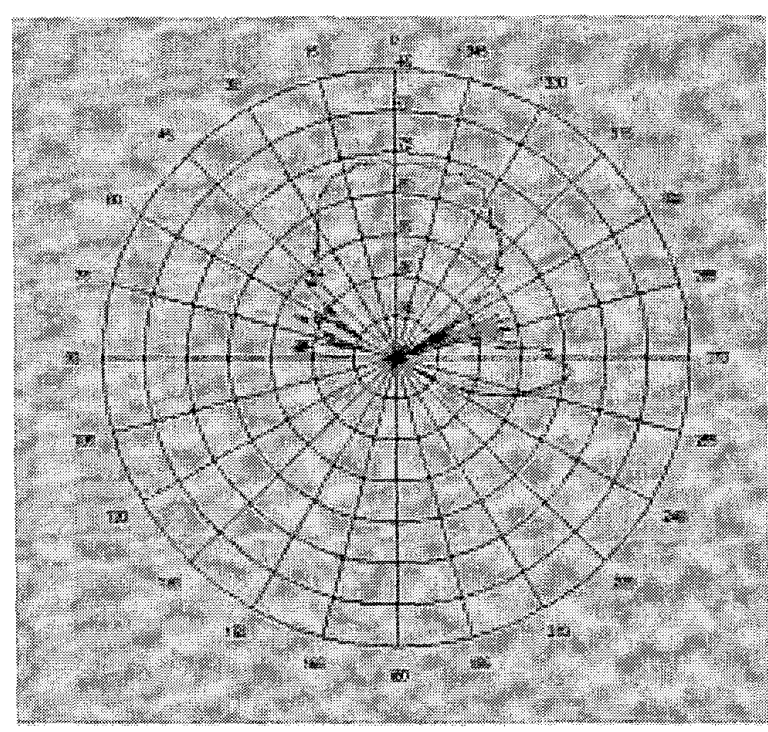

Figure 6. Lend $=2 \mathrm{~mm}, \mathrm{~V}-\mathrm{V}$, In Air

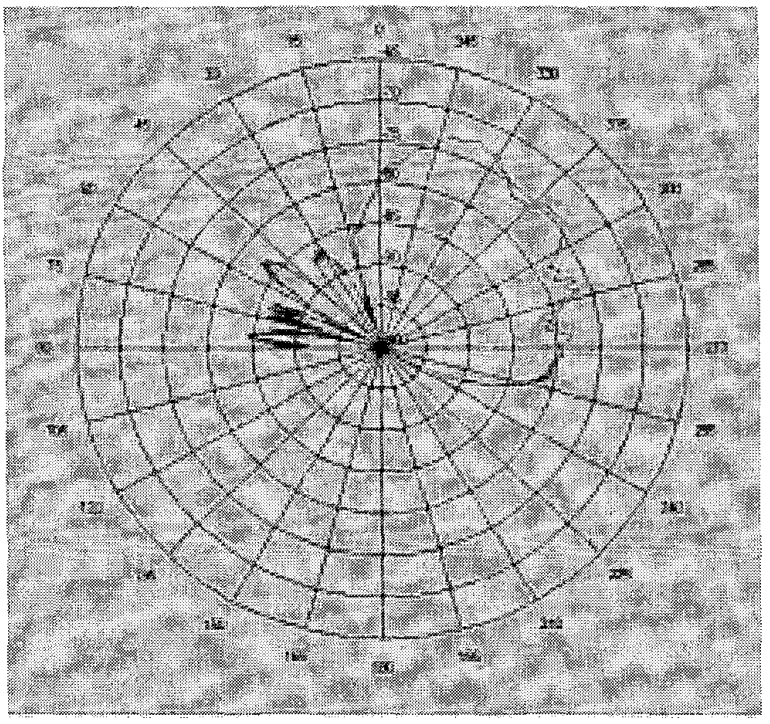

Figure 7. Lend $=2 \mathrm{~mm}$, V-H, In Air

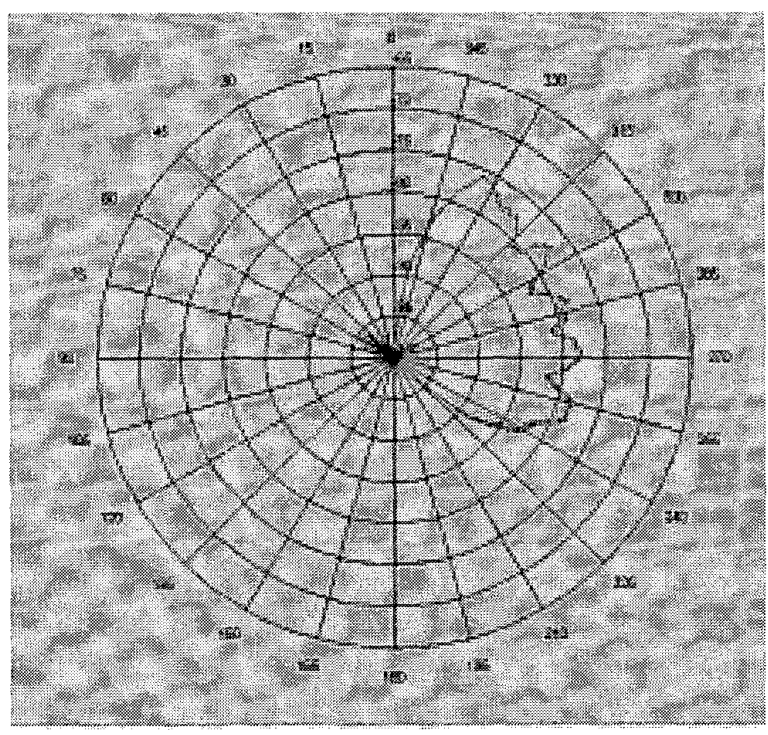

Figure 8. Lend $=2 \mathrm{~mm}, \mathrm{~V}-\mathrm{V}$, On Metal

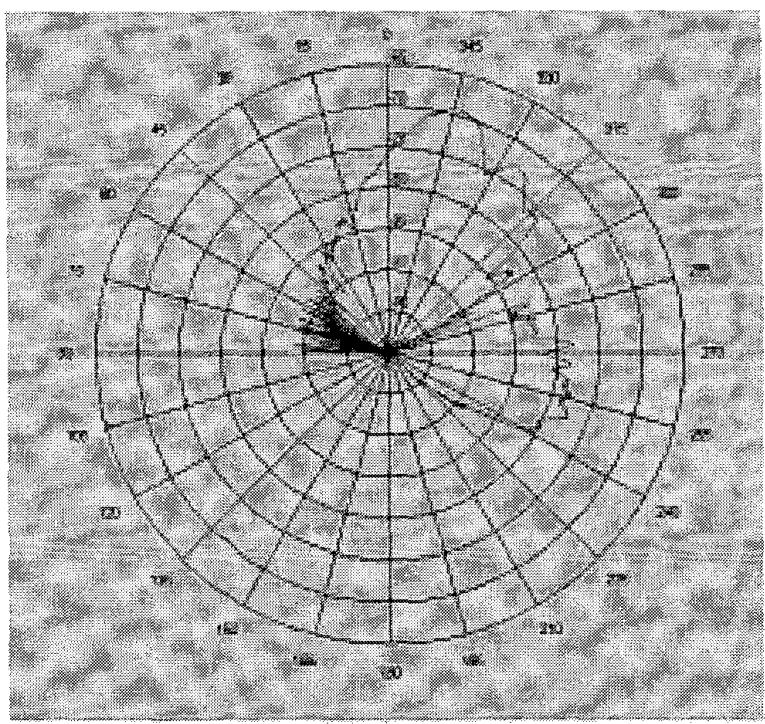

Figure 9. Lend $=2 \mathrm{~mm}, \mathrm{~V}-\mathrm{H}$, On Metal 


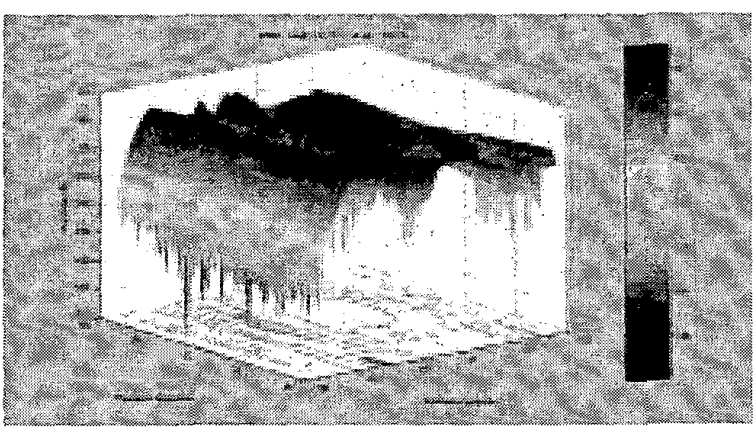

Figure 10. Lend $=2 \mathrm{~mm}, \mathrm{~V}-\mathrm{V}$, In Air

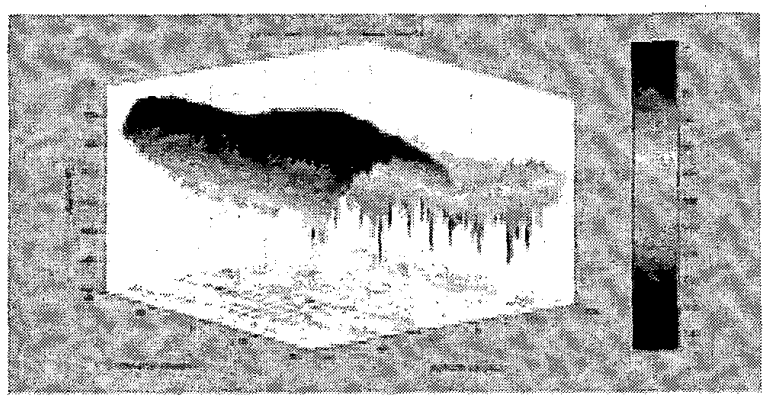

Figure 11. Lend - 2mm, V-V, On Metal

All of the measurements, whether co-polarization, crosspolarization, in air, or on metal, either yielded patterns with extremely wide beamwidths, multiple equal gain lobes, or mainlobes in unexpected directions. Often the cross-polarization patterns were indistinguishable from the copolarization patterns with respect to maximum gain and directivity, although the angles of maximum gain and shape of the patterns may be totally different. Thus, no beamwidth calculations were made from these measurements since the principle plane radiation patterns were so broad.

The patterns of the LTSAs on metal yielded little improvement, but there were several notable differences in the antenna patterns. It should be noted that the copolarization patterns were greatly decreased near endfire, at azimuth $=0^{\circ}$ (refer to Figure 8). Since the radiated electric field is parallel to the mounting plate, the electromagnetic boundary conditions dictate an $\mathbf{E}$ field cancellation near the plate. The cross-polarization field is not restricted in this manner, however note that the cross-polarization pattern actually increased near endfire.

Input VSWR plots for all five designs are shown in Figures 12-16.
LTSA, Lendi=0, VsWR

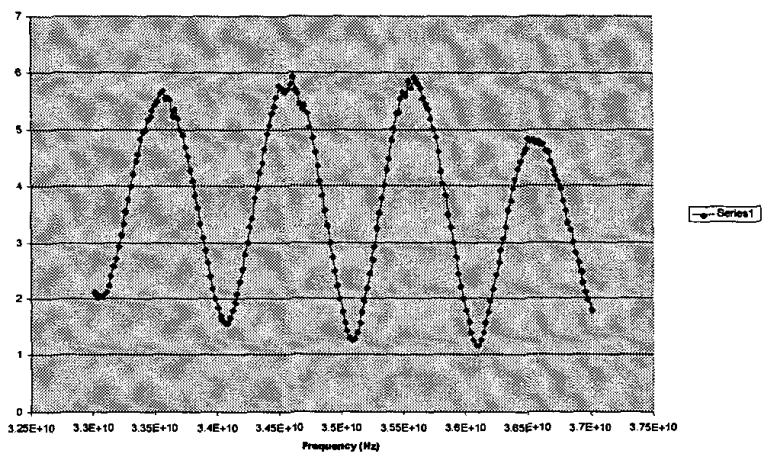

Figure 12. VSWR for Lend $=0$

LTBA, Lend * 1, VBWR

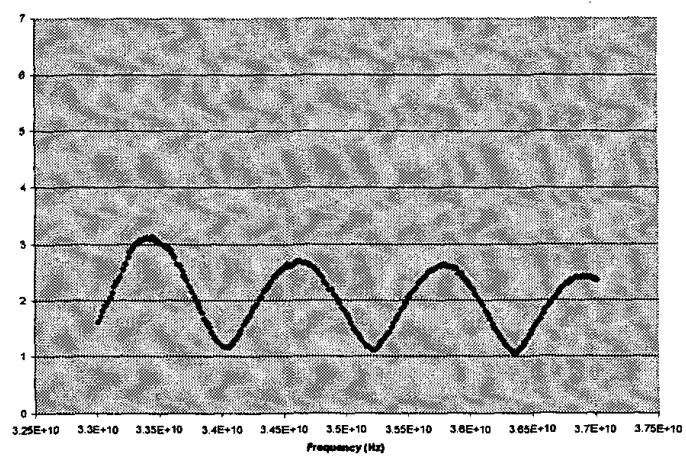

Figure 13. VSWR for Lend $=1$

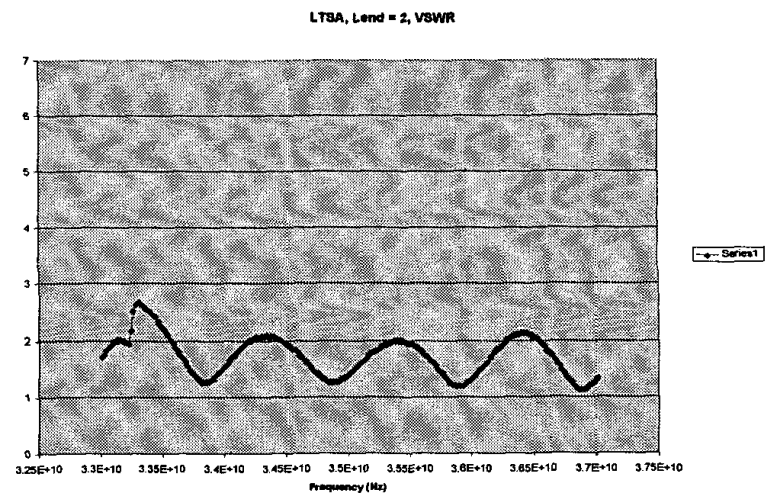

Figure 14. VSWR for Lend $=2$ 


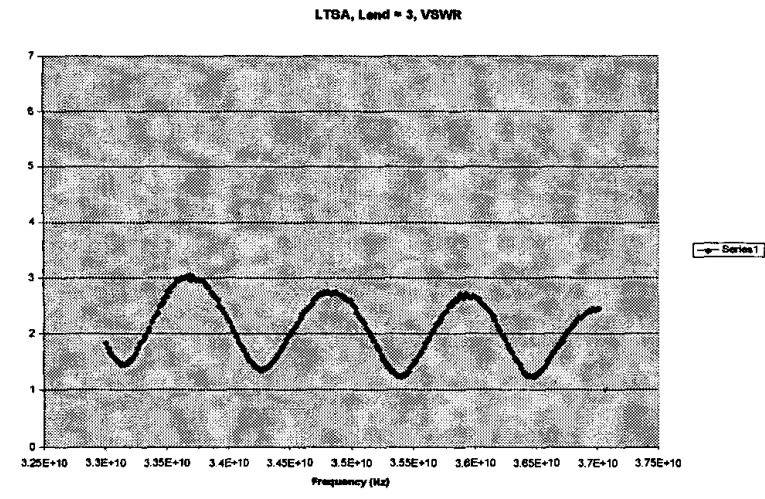

Figure 15. VSWR for Lend $=3$

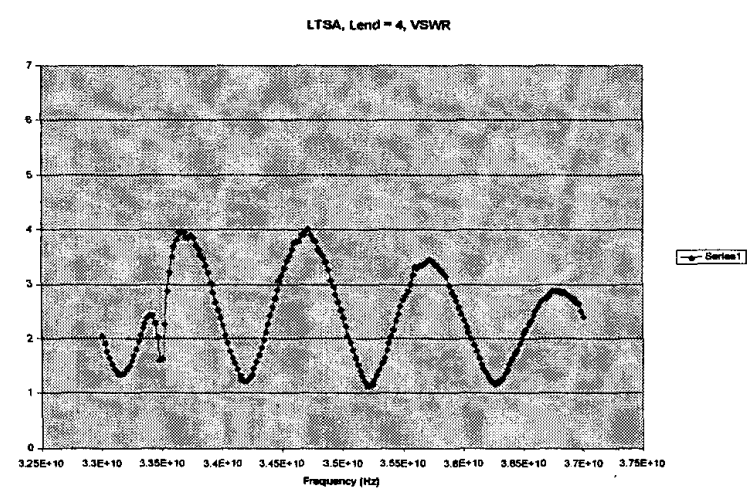

Figure 16. VSWR for Lend $=4$

The network analyzer test port power was $-10 \mathrm{dBm}$ and there were 201 data points over the frequency range of $33 \mathrm{GHz}$ to $37 \mathrm{GHz}$. A network analyzer calibration was performed before the antennas were analyzed. The measurements presented were taken with the antennas held in air. There was almost no change in the VSWR when the antennas were mounted on the aluminum plate. Although the VSWR for the antennas at $35 \mathrm{GHz}$ were almost all below $2.0\left(\mathrm{~L}_{\text {end }}=3\right.$ had VSWR $\approx 2.7$ at $35 \mathrm{GHz}$ ), the plots show a generally poor input impedance match for all designs. The coaxial line feed is the probable source of the impedance mismatch. This mismatch would tend to make these antennas narrow banded and inefficient.

An estimated gain measurement for the LTSA tested with the coaxial feed is $-30 \mathrm{~dB}$. A copy of the gain response is shown in Figure 17. Because of the inefficient feeding method used, these measurements should be interpreted with this issue in mind. It should also be noted that the feed used for the antenna measurements is not a typical feed for use at Ka-band.

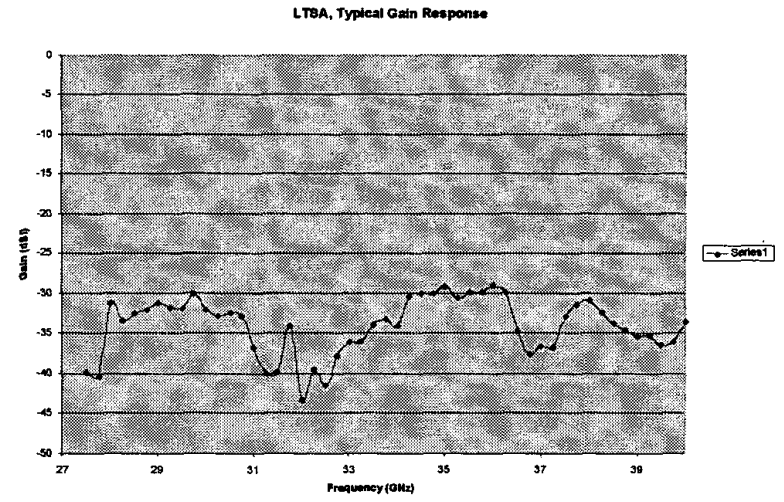

Figure 17. Measured Gain Response, Antenna Main Lobe

\section{Conclusions}

There were differences in the antenna patterns when the two mounting configurations were compared. The boundary conditions for general electromagnetic theory state that the tangential components of the $\mathbf{E}$ field be continuous across any source free conductor/dielectric interface and mounting on a metal plate dictates that the tangential components be zero [3]. It is easy to see from the azimuthal plots that the tangential portion of the $\mathbf{E}$ field near the conducting surface is diminished when the antenna is mounted on the aluminum plate. This causes the pattern at zero degrees to be greatly compromised. Further investigation is needed to determine the minimum antenna to conducting surface separation distance requirement that would restore the antenna pattern near the endfire position.

We believe that the feeding method used in these tests proved to be very inefficient and probably caused some of the additional lobes present in the antenna patterns. In addition, the gain was much lower than expected and this also was most likely caused by the inefficient feed.

\section{REFERENCES}

[1] Smith, Brian J., Linear Tapered Slot Antenna Design for the RIP Fire Projectile, TR-RD-MG-02-05, December 2001.

[2] Lee, Hai Fong and Chen, Wei, Advances in Microstrip and Printed Antennas, pp. 462-463, New York, NY: John Wiley \& Sons, Inc., 1997.

[3] Cheng, David K., Fundamentals of Engineering Electromagnetics, pp. 111-113, Reading, MA: AddisonWesley, 1993. 
Vol. 2-938

\section{BIOGRAPHIES}

Janice C. Rock is a research engineer with the RF Technologies group in the Missile Guidance Directorate, Missile, Research, Development and Engineering Center, US Aviation \& Missile Command; Redstone Arsenal, AL. She has a B.S. in Electrical Engineering from the University of Alabama in Huntsville.

Chris Hamner is a research engineer in radar and electronic systems with the Research, Development, \& Engineering Center of the U.S. Army Aviation \& Missile Command; Redstone Arsenal, AL. He works with airborne seeker simulation, testing, and

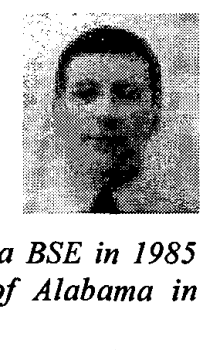
system development. Mr. Hamner received a BSE in 1985 and MSE in 1997 from the University of Alabama in Huntsville (UAH) in Electrical Engineering.

Perry Vanderford is an RF engineer for Simulation Technologies, Inc. in Huntsville, $A L$. He is presently involved in millimeterwave electromagnetic analysis and simulation. He received a B.S.E.E. from Tennessee Technological University and an M.S.E. from the University of Alabama in Huntsville.
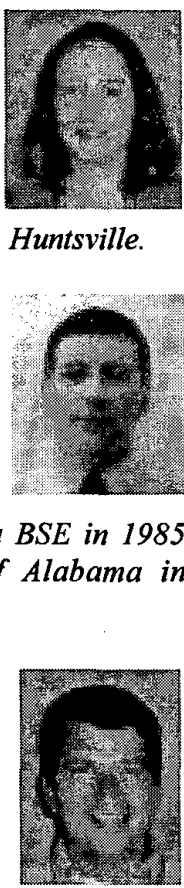